\title{
GENDER-SPECIFIC FEATURES OF ARTERIAL HYPERTENSION DEVELOPMENT AND TREATMENT IN UKRAINE
}

\author{
NATALIIA H. STEPANIUK ${ }^{1}$, NATALIIA I. HUDZ ${ }^{1}$ and ALLA H. STEPANIUK² \\ 'Danylo Halytsky Lviv National Medical University, Lviv, Ukraine \\ ${ }^{2}$ National Pirogov Memorial Medical University, Vinnytsya, Ukraine
}

\begin{abstract}
Modern cardiology, as well as other fields of medicine, focuses particularly close attention on the gender aspects of prevention, diagnosis, and treatment, as well as the social significance of cardiovascular diseases since they are the leading cause of mortality among both men and women, but the proportions of women dying from the circulatory system diseases are significantly higher than those for men. Currently available guidelines for the arterial hypertension treatment recommend the same approaches to the treatment of hypertensive patients, regardless of their gender. However, some studies specify the differences between men and women in controlling blood pressure and emphasize the need for different approaches to treating female and male hypertensive patients, taking into account gender features of the disease development.
\end{abstract}

Keywords: arterial hypertension, gender differences, sex hormone, menopause

Cardiovascular diseases (CVDs) are recognized to be major health and social problems in most developed countries in the world, including Ukraine. Hypertension (HTN) or high blood pressure (HBP) accounts for the biggest share of cardiovascular morbidity. HTN is known to be the major risk factor and one of the leading causes of the working-age population's death and disability (1-5). According to statistics from the National Scientific Center, M. D. Strazhesko Institute of Cardiology of the National Academy of Medical Sciences of Ukraine $(6,7)$, the share of arterial HTN in the total CVDs burden in Ukraine constitutes $41.2 \%$. Furthermore, Ukraine has recorded a markedly increased proportion of cardiovascular (CV) and cerebrovascular diseases in the total burden of diseases, which has lately risen by $27 \%$ for men, whereas the corresponding statistics for women has been $33 \%$. HBP accounts for about $50 \%$ of the burden of these diseases, which requires that the population-based and individual strategies for their prevention and treatment should be immediately implemented. Moreover, $26.6 \%$ of the adult population of the world had HTN in 2000 (957-987 million). It was expected that in 2025 the number of arterial HTN patients in the world will increase by $60 \%$ and reach 1.56 billion people that will constitute $29.2 \%$ of the adult population (8-10).
$30 \%$ of the Ukrainian adult population suffered from HTN in 2014 (9).

The incidence of arterial HTN is known to vary with age with considerable gender difference in the chances of being affected by hypertensive diseases: the ratio for women reporting about arterial HTN condition increases with aging $(1,5)$. Whereas this type of $\mathrm{CV}$ pathology prevalence is higher for males aged under 60 than for females, the situation becomes reversed after 60 years of age $(1,5,10)$. Arterial HTN is a major risk factor for the CVDs and kidney disease development, having a negative impact on life quality and increasing in postmenopausal women mortality $(1,2,8,9)$.

Modern cardiology, as well as other fields of medicine, focuses particularly close attention on the gender aspects of prevention, diagnosis, and treatment, as well as the social significance of CVDs (14, 8-12). CVDs are the leading cause of death among both men and women, accounting for onethird of all deaths in the world (8). However, the proportions of women passing away from diseases of the circulatory system are significantly higher than those for men (2,13-16). Thus, according to the data released in 2004 by World Health Organization, the mortality rate of European women from CVDs is $55 \%$, while the mortality rate for men

* Corresponding author: e-mail: natali.stepanuyk@gmail.com 
is only $43 \%$ (16). However, N. Townsend et al. (2015) state that the death rate from CVDs for women aged under 65 is $26 \%$, whereas the mortality rate for men is $30 \%$, but the percentage number of women aged under 75 who die from this pathology increases and totals $36 \%$, while the rate for men of the same age is $35 \%$ (17). In general, CVDs account for $40 \%$ of all deaths among men and $49 \%$ of all deaths among women in Europe (17). Moreover, in Ukraine, the mortality rate for coronary heart disease (CHD) for 55-60-year-old people is greater compared to the equivalent rate in France for people 20 years older (18).

Experts attribute this fact to the lack of deep awareness that CVD in women emerges to be an acute problem, which calls for a gender-oriented approach to the study of arterial HTN, in particular, with the aim of reducing in CVD incidence and complications as well as improving patients' quality of life $(1,2,19)$.

Although the mechanisms responsible for gender differences in blood pressure (BP) control have not been clearly determined yet, there is substantial evidence that sex hormones play a crucial role in gender differences as they are involved in BP regulation $(2,15,19,20)$.

One of the main reasons for a considerable increase in the prevalence of HTN in women aged over 60 is the onset of menopause caused by estrogen deficiency $(2,19)$. However, Reckelhoff states that deficiency of female hormone may not be one contributing factor for the increased BP (15).

On the one hand, estrogen deficiency in the postmenopausal period is regarded as a natural physiological process, on the other one, it can create risks for the development of various disorders. Estrogens are vasoactive substances that affect all layers of the arterial wall of resistive vessels, reducing BP $(2,19)$. Estrogens exert endotheliumdependent and endothelium-independent vasodilator effects (19). Therefore, endothelium-dependent vasodilation declines with the onset of menopause that results from a decrease in nitric oxide (NO) production $(15,19)$. In addition, estrogen deficiency leads to vasoconstriction by different mechanisms (15). The renin-angiotensin system and NO are the two major regulators that modulate BP levels as well as the reabsorption and excretion of sodium $(19,21)$. Estrogens affect this procedure by regulating NO levels as well as different angiotensin receptor expression levels. Such positive effects of estrogens are lost when menopause starts, which causes an increase in BP $(15,19,21,22)$.
However, according to research findings of the Ukrainian scientists, women approaching menopause are more often diagnosed with the development of renovascular HTN as a consequence of atherosclerotic renal artery stenosis. Thus, approximately $50 \%$ of postmenopausal female patients are diagnosed with a sodium-dependent form of arterial HTN (19).

An increased BP in the postmenopausal period may also be conditioned by the accumulation of visceral adipose tissue and it is regarded as a component of menopausal metabolic syndrome (MBS) $(19,22)$. In turn, hyperinsulinemia which accompanies menopausal MBS contributes to the development of arterial HTN as it causes enhanced renal sodium reabsorption, intracellular fluid retention, elevated sodium and calcium concentrations in the arteriolar vascular smooth muscle cells, and their hypersensitivity to pressor agents. Postmenopausal women are reported to face insulin resistance, which is a component of menopausal MBS and, above all, is caused by stimulation of the sympathetic nervous and renin-angiotensin systems, impaired lipid metabolism in the form of atherogenic dyslipoproteinemia and obesity as well as activation of pro-inflammatory cytokines (19). Thus, insulin resistance is a key driving factor for the development of disorders in carbohydrate, purine and lipid metabolism, blood coagulation involved in the pathogenesis of arterial HTN in women during this life period $(19,21)$.

According to Wassertheil-Smoller et al. (23) and Zhuravlyova et al. (19), the prevalence of arterial HTN in women depends on age, body weight, race, and smoking. The prevalence of HTN among women aged $50-59$ is $26.7 \%$, aged $60-69$ is $41 \%$, and aged $70-79$ is $53.4 \%$ (23).

The effectiveness of antihypertensive therapy for women over 50 decreases from $20 \%$ to $8 \%$. This, in turn, increases the risk of CHD development by 3 times, whereas the stroke development risk rises by 7 times. With each subsequent decade of life, the incidence of death of women from CVDs increases by 3-5 times (24). At the same time there are prerequisites for the progression of HTN after menopause: endothelial dysfunction, obesity, increased sympathetic nervous system activity, activation of the renin-angiotensin-aldosterone system, and hypersensitivity to sodium chloride $(2,3,15,19$, 24). These disorders lead to increased arteriole tone, vascular remodeling, increased vascular resistance, and HBP. Decreased estrogen levels are the cause of dyslipidemia and carbohydrate metabolism disorders (24). 
Therefore, the above-mentioned data reveal particular features of distinguishing the development and progression of arterial HTN in females in comparison with males due to both hormonal and age-related factors, which determined the relevance of writing this review article.

\section{Aim of the study}

The aim of the given study is to determine the peculiarities of arterial HTN development and progression and to assess the gender-related differences in its treatment of the Ukrainian population on the basis of the analysis of research data and statistics from Medline, Scopus, Web of Science Core electronic databases of 2000-2019.

\section{RESULTS OF STUDY}

Currently available guidelines for arterial HTN treatment recommend that the same approaches to treat hypertensive patients, regardless of their gender. However, some studies specify the differences between men and women in controlling BP and emphasize the need for a different approach to treat female and male hypertensive patients, taking into account gender features of the disease development $(2,3,15,19,23,24)$.

Amosova et al. identified gender-specific features of antihypertensive therapy involving 421 both male and female patients with uncomplicated arterial HTN treated with the fixed-dose combination of perindopril and amlodipine. It was found that the target optimal office BP level was reached in $80.5 \%$ of the patients in 6 months. However, the rate of optimal in-office $\mathrm{BP}$ regulation was better among women compared to men (1). These data are in compliance with epidemiological studies conducted in Ukraine: women demonstrate better BP control than men with the corresponding proportions of $25 \%$ for female patients and $10 \%$ for male ones among the urban population and those of $15 \%$ for female persons versus $3 \%$ for male ones among the rural population (25). However, some authors revealed better BP control in male patients among 9821 totally observed patients with arterial HTN, despite better adherence in females (3). Therefore, the authors emphasize that in the future measures aimed at improving BP control for hypertensive patients should be different for men and women (19, 24, 26).

The 2007 ESC/ESH European guidelines for the management of arterial hypertension developed jointly by the European Society of Cardiologists and the European Society of Hypertension and issued in
2007 provide principal recommendations to be taken into account in the determination and implementation of diagnostic or therapeutic strategies to manage arterial HTN in women. These principles are the result of a joint work of professionals involved in the Task Force group headed by Giuseppe Mancia (University of Milano-Bicocca, Milan, Italy) from ESH and Guy De Backer (University of Ghent, Gent, Belgium) from ESH. According to these authors, standard guidelines for arterial HTN management should be followed when treating menopausal female patients with arterial HTN (5, 26). The most important recommendations are associated with avoiding the administration of potentially teratogenic medicinal products (angiotensin-converting enzyme (ACE) inhibitors and angiotensin receptor blockers) in fertile women. If they were nevertheless prescribed to a woman of childbearing age, the medications should be immediately canceled in the event of pregnancy $(5,26)$.

However, the 2018 ESC Guidelines (27) provide principal rules and regulations on the implementation of the approaches to the treatment of arterial HTN in pregnant women. Arterial HTN during pregnancy is the leading cause of complications and mortality among 5 to $10 \%$ of pregnant women worldwide. The maternal risks are stroke, multiple organ failure, and disseminated intravascular coagulation. The fetus has the risk of intrauterine growth retardation, prematurity, or prenatal death. Therefore, ESC specialists recommend that pregnant women at high and very high risk of preeclampsia administer low-dose aspirin (100-150 $\mathrm{mg}$ ) daily starting from 12 to 36 weeks of pregnancy. The purpose of the drug treatment for arterial HTN in pregnancy is to reduce the risks for both mother and her fetus. According to the guidelines, the drug therapy of pregnant women is recommended to start in the incidence of a steady increase in BP above 150/95 $\mathrm{mm} \mathrm{Hg}$. Women with gestational HTN with or without proteinuria should receive treatment with antihypertensive drugs at a BP level of 140/90 mm Hg or above. The range of medications for the arterial HTN treatment during pregnancy includes methyldopa, labetalol, and calcium channel blockers. ACE inhibitors and angiotensin II receptor blockers (ARBs) are contraindicated in pregnant women because of adverse effects on both fetus and newborn. According to the guidelines, the aim for the target BP level in pregnant women is below 140/90 mm Hg (27, 28).

A particular form of symptomatic HTN in women is HTN which results from the use of combined oral contraceptives (estrogen-progesterone- 
containing medicines) $(5,27,28)$. According to the study (29) focusing on the evaluation of the relationship between oral contraceptive use and arterial HTN development, the administration of birth control drugs was statistically significant as contributing to an increased systolic and diastolic BP ( $\mathrm{p}<$ 0.001 ). This study involved 3356 Korean women aged 35-55 years old within the period of 20072009. It was revealed that both systolic and diastolic BP levels were higher among women who had been using oral contraceptives for more than 24 months compared to women who had never administered them (29).

Research data indicate $(5,27-29)$ that even low estrogen-containing oral contraceptives increase the risk of HTN, stroke, and myocardial infarction. Therefore, hypertensive women are advised to use single-phase progestin-only drugs as appropriate options for contraception whereas keeping in mind that their cardiovascular effects have not yet been extensively studied. Particular risk categories are overweight women aged over 35, and smokers. Alternative contraceptive methods are recommended if several of these risk factors are combined (5). Patients with arterial HTN caused by hormonal contraception are recommended to stop using estrogen-containing medicines and switch to other contraceptive methods (27). However, women with well-controlled arterial HTN may take oral contraceptives, but monitoring their BP levels should be under strict control. Under such conditions, women who take contraceptive drugs are advised to use diuretics as antihypertensive drugs since an increase in BP is accompanied by fluid retention (5).

Atherosclerosis or fibromuscular dysplasia of renal arteries most often induce HTN and renal disease (30). Menopause increases the risk of renal disease and glomerulosclerosis. The kidneys play important role in the water homeostasis regulation and, therefore, controlling BP, and protective effects of estrogens in renal HTN (31). In premenopausal women, cases of arterial HTN with fibromuscular dysplasia of renal arteries (one of the most common causes of renal vascular HTN) are quite common compared to men. Fibromuscular dysplasia most often affects women under 50 years. In such cases, the preferred therapeutic measures for HTN treatment can be calcium channel blockers. ACEinhibitor and ARBs therapy may lead to serum creatinine elevation and hyperkalemia. Therefore, the main treatment method for this type of secondary arterial HTN is surgery (30).
According to the opinion of ESC experts (27), there is no conclusive evidence that hormone replacement therapy in menopausal women can lead to a dramatic increase in BP. Thus, it can be assumed that hormone replacement therapy is not contraindicated in women with HTN, as long as BP can be controlled by the antihypertensive drugs administration.

\section{CONCLUSIONS}

Thus, according to the existing research data, there are no specific recommendations on employing particular strategies to treat arterial HTN in female and male patients. However, the present study analysis reveals clear differences in the mechanisms of hypertension development in women compared to men, as well as emphasizes the gender-specific differences in administering BP control. Therefore, this study results stress the need for further research into gender specificity of arterial HTN treatment to determine the most effective approaches stimulating arterial HTN risk reduction and its further complication prevention for men and women.

\section{Conflicts of interest}

The authors declare no conflict of interest.

\section{REFERENCES}

1. Amosova K.M., Rudenko Ju.V.: Medical Perspectives 21, 31 (2016).

2. Kovalenko V.M., Dolzhenko M.M., Nesukay E.G., DiachenkoYa.S., Nudchenko O.O., Faraj K.: Ukrainian J. Cardiology 4 (Suppl. 3), 15 (2016).

3. Radchenko G.D., Slascheva T.G., Sirenko Yu.M., Mushtenko L.O.: Ukrainian J. Cardio$\operatorname{logy} 4,34$ (2015).

4. Baggio G., Corsini A., Floreani A., Giannini S.: Clin. Chem. Lab. Med. 51, 713 (2013).

5. Barna O.M., Hrebennyk M.V., Portna O.I., Karapetian O.A.: Medicine of Ukraine 3, 9 (2010).

6. Dorogoy A.P., Kirichenko A.G., Revenko I.L., et al.: Stress and diseases of the circulatory system, Kyiv 2015.

7. Kovalenko V.M., Kornatsky V.M.: Dynamics of health status of the people of Ukraine and regional features, Kyiv 2012.

8. Jha V., Wang A.Y., Wang H.: Nephrol. Dial. Transplant. 27, Suppl. N 3, 32 (2012). 
9. Lashkul Z.V.: Modern Medical Technologies 2, 134 (2014).

10. Kearney P.M., Whelton M., Reynolds K., Muntner P., Whelton P.K., He J.: Lancet 365, 217, 2005.

11. Kochilas L.K., Vinocur J. M., Menk J.S.: J. Am. Heart Assoc. 3, 1 (2014).

12. Carrero J.J., Hecking M., Chesnaye N.C., Jager K.J.: Nat. Rev. Nephrol. 14, 151 (2018).

13. Bugaenko V.V.: Ukrainian J. Cardiology 6, 100 (2015).

14. Davidova I.V.,Mymrenko S.N., Perepelchenko N.A., Konoplianik LI.: J. Womans Health 4, 43 (2016).

15. Reckelhoff J.F.: Hypertension 37, 1199 (2001).

16. Stramba-Badiale M., Fox K.M., Priori S.G., Collins P., Daly C., et al.: Eur. Heart J. 27, 994 (2006).

17. Townsend N., Nichols M., Scarborough P., Rayner M.: Eur. Heart J. 36, 2696 (2015).

18. Nichols M., Townsend N., Scarborough P., Rayner M.: Eur. Heart J. 35, 2950 (2014).

19. Zhuravlyova L.V., Butova T.S.: Medicines of Ukraine 5-6, 4 (2016).

20. Maranon R., Reckelhoff J.: Clin. Sci. (Lond) 125, 311 (2013).
21. Lima R., Woffort M., Reckelhoff J.F.: Curr. Hypertens. Rep. 14, 254 (2012).

22. Katholi R.E., Couri D.M.: Int. J. Hypertens. 2011, 10 pages (2011).

23. Wassertheil-Smoller S., Anderson G., Psaty B., Black H.R., Manson J., et al.: Hypertension 36, 780 (2000).

24. Zhdan V.M., Kitura Ye.M., Kitura O.Ye., et al.: Fam. Med. 2, 76 (2019).

25. Gorbas I.M.: Ukrainian J. Cardiology 2, 21, (2007)

26. Mancia G., Backer G., Anna Dominiczak A., Cifkova R., Fagard R., et al.: J. Hypertens. 25, 1105 (2007).

27. Williams B., Mancia G., Spiering W., Rosei E.A., Azizi M., et al.: J. Hypertens. 36, 2284 (2018).

28. Braunthal S., Brateanu A.: SAGE Open Med. 7 , 1 (2019).

29. Park H., Kim K.: BMC Womens Health 13, 39 (2013).

30. Bokhari M.R.: Renal Artery Stenosis Treasure Island, 2018.

31. Barton M., Meyer M.R.: Hypertension 54, 11 (2009).

(C) 2020 by Polish Pharmaceutical Society. This is an access article under the CC BY NC license (c) (1) (9) (http://creativecommons.org/licenses/by-nc/4.0/). 